\title{
Multimodal treatment of myophasal chronic pelvic pain in women: institutional experience
}

\author{
Guadalupe Guerrero Reyes ${ }^{1 *}$, Cristobal Barragán Ochoa ${ }^{1}$, Claudia M. Moysen Marin ${ }^{1}$, \\ Vaney B. Ayala Quispe ${ }^{1}$, Laura Escobar Del Barco', Adrian Gutiérrez González², \\ Aldo García-Bailón ${ }^{2}$, Ricardo Hernandez Velazquezz ${ }^{1}$
}

\begin{abstract}
${ }^{1}$ Department of Gynecological Urology, The National Medical Center, 20 de Noviembre, CMN 20 NOV, Mexico
${ }^{2}$ Department of Urology, The University Hospital, José Eleuterio González, Monterrey NL, Mexico.
\end{abstract}

Received: 02 March 2019

Accepted: 02 April 2019

*Correspondence:

Dr. Guadalupe Guerrero Reyes,

E-mail: barraganc511@gmail.com

Copyright: (c) the author(s), publisher and licensee Medip Academy. This is an open-access article distributed under the terms of the Creative Commons Attribution Non-Commercial License, which permits unrestricted non-commercial use, distribution, and reproduction in any medium, provided the original work is properly cited.

\begin{abstract}
Background: Chronic pelvic pain of myofascial origin is an underdiagnosed condition and with partial responses to the different treatment modalities. The objective of this study was to report the results of the multimodal management of Myofascial Chronic Pelvic Pain.

Methods: Retrospective cohort of 33 patients with myofascial chronic pelvic pain, during the period 01 January 2016 to August 31, 2018, who were treated based on trigger point infiltration and analgesic vaginal electrostimulation. The analogous visual pain scale (VAS) was used at the beginning, sixth, twelfth and six months after the last electrostimulation session, in order to measure the effect of the treatment.

Results: During the study period, 32 patients with chronic myofascial pelvic pain were found who received the multimodal scheme that is offered in our clinic. The average score of the EVA before starting the treatment was 8.5 points, at the sixth session of the treatment the average EVA was 3.6 points, at the twelfth session of 1.3 points and the average score of the EVA at six months after the last session of the multimodal treatment was 2.9 points. A significant difference was demonstrated in the average EVA score in the first with respect to the sixth, twelfth and six months $(\mathrm{p}=<0.000)$. There were no complications or adverse effects secondary to the multimodal treatment.

Conclusions: Multimodal treatment was associated with progressive improvement in $80 \%$ of patients with miofascial chronic pelvic pain.
\end{abstract}

Keywords: Chronic myofascial pelvic pain, Chronic pelvic pain, Myofascial syndrome, Pelvic floor hypertonic, Pelvic floor spams

\section{INTRODUCTION}

Chronic pelvic pain (PCD), affects $16 \%$ of women in the United States, being the myofascial origin the most frequent cause with a prevalence of 9 to $24 \%$, in our country there are no statistical data of this condition. ${ }^{1,2}$ It is a complex health problem that affects the quality of life of patients with various urogynecological disorders, taking them in the vast majority of cases to depression, anxiety and fatigue. ${ }^{3}$ The etiology so far is unknown, some theories relate to muscle fiber damage which causes contraction of the pelvic floor muscles causing hypoxia, inflammatory mediators production and as a consequence the generation of myofascial pain. ${ }^{4,5}$ It is now known that $40 \%$ of diagnostic laparoscopies are attributed to myofascial syndrome (MS). In the absence of 
demonstrable organic pathology and persistence of pelvic pain for more than six months, MS is the most frequent etiologic cause. The clinical presentation is very broad, from lower urinary tract symptomatology, sexual dysfunction, rectal, to being a disabling pain. ${ }^{6}$

The diagnostic approach of this condition is challenging, from the gynecological evaluation of the patients, to the lack of clinical suspicion on the part of the medical group of the MS, as a consequence a poor diagnosis is made, and the treatment fails, perpetuating the chronicity of the disease. $^{7}$

There are several modalities of treatments, which must be staggered according to the recommendations of the American pain association, often poor clinical responses are obtained, so the management must be multidisciplinary. ${ }^{8}$ The present study was conducted in order to report our results of multimodal management in patients with chronic pelvic pain secondary to myofascial syndrome, in the Clinic of Gynecological Urology of the "Centro Médico Nacional 20 de Noviembre, ISSSTE, Mexico".

\section{METHODS}

A retrospective cohort of patients with chronic myofascial pelvic pain was performed, in order to report our results of multimodal treatment which consisted of: Infiltration of painful trigger points with $1 \mathrm{~cm} 3$ of the dilution of dexamatasone $8 \mathrm{mg}$, bupivacaine $0.25 \%$ and subsequently 12 sessions of vaginal endocavitary analgesic electrostimulation initially at high frequencies and later at medium frequencies, the electrostimulation sessions were performed 3 times a week.

The response to the treatment was made by means of the Visual Pain Scale (VAS), applying it before starting the treatment, sixth, twelfth and six months after the last session of electro-vaginal stimulation.

The study period was from January 01, 2016 to August 31,2018 . Only patients who were the cause of chronic pelvic pain were included in the myofascial, who completed the multimodal treatment scheme and had six months follow-up concluded the multimodal scheme, excluding those with other causes of chronic pelvic pain.

\section{Statistical analysis}

The data was recorded in a pre-established format and subsequently analyzed using the SPSS Statistics V25.0 program. We calculated means, standard deviations, averages of demographic variables, such as: age, body mass index, births, type 2 diabetes mellitus, neurological disease, rheumatology, and sexual abuse. To establish whether there is a significant difference between the initial, sixth, twelfth and six months EVA after the last multimodal treatment session, the Wilcoxon rank test was used.

\section{RESULTS}

Authors analyzed 9,206 consultations from January 01, 2016 to August 31, 2018, of which myofascial pelvic pain was presented only in 33 women $(0.35 \%)$, which are included in this study. The average age was $52.7 \pm 10.2$ years. In the 33 patients they had a history of having applied at least one unsuccessful treatment strategy, usually analgesics in combination with anxiolytics. 55\% had at least 3 pregnancies and $45 \%$ had more than 4 pregnancies. $30 \%$ of women had a history of 2 caesarean sections. Other demographic characteristics in (Table 1).

Table 1: Clinical and demographic characteristics of the study population.

\begin{tabular}{|c|c|c|}
\hline Characteristics & & $\begin{array}{l}\text { Patients } \\
n=33\end{array}$ \\
\hline Gende & Female & 33 \\
\hline Age & Average \pm SD* & $52.7 \pm 10.2$ \\
\hline Body mass index & Average \pm SD & $27.4 \pm 10.2$ \\
\hline \multirow[t]{3}{*}{ Occupation } & Hausewife & (11) $36 \%$ \\
\hline & Office work & (17) $51 \%$ \\
\hline & Others & (05) $13 \%$ \\
\hline \multirow[t]{2}{*}{ Pregnancies } & $\leq 3$ & (19) $55 \%$ \\
\hline & $\geq 4$ & (14) $45 \%$ \\
\hline \multirow[t]{2}{*}{ Births } & $\leq 3$ & (23) $69 \%$ \\
\hline & $\geq 4$ & (10) $30 \%$ \\
\hline \multirow[t]{2}{*}{ Cesareans } & $\leq 2$ & (10) $30 \%$ \\
\hline & $\geq 3$ & (23) $0 \%$ \\
\hline \multirow[t]{2}{*}{ Abortions } & $\leq 3$ & (01) $3 \%$ \\
\hline & $\geq 4$ & (00) $0 \%$ \\
\hline Forceps & Antecedent & (01) $4 \%$ \\
\hline \multirow[t]{3}{*}{ Diabetes } & $\begin{array}{l}\text { Without } \\
\text { diabetes }\end{array}$ & (21) $72 \%$ \\
\hline & $<10$ años & (07) $15 \%$ \\
\hline & $>10$ años & (05) $13 \%$ \\
\hline $\begin{array}{l}\text { Rheumatologic } \\
\text { disease }\end{array}$ & Diagnosed & (07) $15 \%$ \\
\hline Neurological disease & Diagnosed & (04) $12 \%$ \\
\hline Sexual abuse & Antecedent & $4 \%$ \\
\hline
\end{tabular}

*DE: Standard deviation

In Table 2, the results of multimodal therapy are shown in the 33 patients. The initial score was 8.6, after the treatment at the sixth session an improvement of $50 \%$ was presented, bringing the EVA to 3.5 , in the twelfth session to 1 point and to the six months after the last electrostimulation session of 2.94 in the $100 \%$ of patients.

When applying the Wilcoxon rank test, a significant difference is shown between the average score evaluated in the first session before applying the multimodal treatment with respect to the average VAS score in the sixth, twelfth and six months after the last multimodal treatment session $(\mathrm{p}=<0.00)$. 
Table 2: Average score of the application of the visual analogous pain scale (VAS).

\begin{tabular}{|c|c|c|c|c|c|}
\hline & \multicolumn{2}{|c|}{ Descriptive statistics } & \multicolumn{3}{|c|}{ Wilcoxon range tests } \\
\hline VAS evaluation & $\mathrm{N}$ & VAS Average* & $\begin{array}{l}\text { VAS Sixth session } \\
- \\
\text { VAS First session }\end{array}$ & $\begin{array}{l}\text { VAS Twelfth session } \\
- \\
\text { VAS First session }\end{array}$ & $\begin{array}{l}\text { VAS } 6 \text { Months } \\
- \\
\text { VAS First session }\end{array}$ \\
\hline VAS First session & 33 & 8.64 & & & \\
\hline VAS Sixth session & 33 & 3.52 & $-5.145(.000 * *)$ & & \\
\hline VAS Twelfth session & 33 & 1.39 & & $-5.088(.000 * *)$ & \\
\hline VAS Six months & 33 & 2.94 & & & $-5.055(.000 * *)$ \\
\hline
\end{tabular}

\section{DISCUSSION}

Myofascial syndrome is a hypertonic disorder of the pelvic floor that can present with pain symptoms or pelvic floor dysfunction. ${ }^{9}$ The pain is often vague and poorly localized, described as painful, throbbing, feeling pressure or heaviness, vaginal, rectal or suprapubic, in one or both lower quadrants. ${ }^{10}$ It usually gets worse as the day progresses and usually with the pelvic floor muscle activities, such as sitting, walking, exercising, having sex, urination or evacuation. A classic symptom is pain after sexual activity that can exacerbate symptoms 12 to 48 hours after intercourse. ${ }^{11}$

Other causes of chronic pelvic pain muss be discard, the diagnosis is made by palpating trigger points or by noticing the hypertonicity of the pelvic floor muscles. ${ }^{12}$ Part of the problem of the diagnosis of this disease is that it is not first suspected and first-contact physicians generally do not perform the exploration of the pelvic floor muscles. ${ }^{13}$

Chronic pelvic pain is a complex condition where monomodal treatment options do not provide a significant improvement in the control of symptoms. ${ }^{14}$ Multiple treatments have been tried from pharmacological conservatives to invasive all with partial responses or with important side effects, in our study we focus on multimodal treatment. ${ }^{15}$ Multimodal treatment, which is defined as the intervention of two or more therapies with a single objective, is imperative in the management of this disease. ${ }^{16}$

The pain-generating muscles by central or peripheral sensitization remain active, here pharmacological therapy with a focus on muscle relaxation helps maintain this muscle inactivation that leads to the neurochemical cascade that keeps the muscles in a constant hypertonic state, but the single application of this pharmacological therapy is less beneficial than the combination with other direct interventions. ${ }^{17}$

Among the complications reported with the injection of trigger points with wet technique are puncture site hematoma, infection and adverse drug reaction, which can be prevented by applying pressure after each infiltration for 1 minute, negative vaginal cultures and adequate technique. asepsis and antisepsis as well as treatment of any allergic event respectively. The importance of identifying and treating the hypertonic component of the pelvic floor allows better control and prevents the perpetuation of the disease and improving the quality of life of patients. ${ }^{18,19}$

The vaginal electro stimulation at high and medium frequencies has an analgesic effect which is achieved by producing heat over the application area, decreasing local inflammation mediators, which, as we know, play an important role in the pathophysiology of the myofascial syndrome. He attributes that by direct stimulation on the nerve terminals it causes desensitization, thereby reducing the painful stimulus. ${ }^{18}$

There is little medical evidence about the use of analgesic electro-stimulation for chronic pelvic pain, and much less in the application of myofascial pain of the pelvic floor with endocavitary catheter. Most of the literature focuses on the use of this treatment in urinary incontinence. ${ }^{19}$

The first study showing the use of vaginal transcutaneous electrical nerve stimulation was by Nappi E. and colleagues, who recruited 29 patients with dyspareunia and vaginismus, who underwent electro-stimulation with a vaginal probe, a weekly session to complete 10 sessions. ${ }^{20}$ The parameters used for electrostimulation were: biphasic current of frequency $1-4 \mathrm{~Hz}$, pulse width $0.1-0.3 \mathrm{~ms}$ and intensity between $0-70 \mathrm{~mA}$. Their results showed significant improvement of the EVA, achieving that the vast majority of patients return to sexual activity, however, their follow-up period was very short of one month.

De Bernardes and colobaradores, compared the use of vaginal electro-stimulation against placebo in 26 patients with chronic pelvic pain, showed that the patients submitted to vaginal electrostimulation presented at the end of the study $80 \%$ improvement compared to $56.5 \%$ of the patients who received placebo, concluding that vaginal electrical stimulation was more effective than placebo for the control of chronic pelvic pain. ${ }^{21}$ 
This same author carried out another study where he evaluated the effectiveness of electro-stimulation with a vaginal probe in 24 women with chronic pelvic pain, who underwent vaginal analgesic electro-stimulation with parameters that were adjusted according to the tolerance of the patient, with sessions of 30 minutes 2 to 3 times per week managing to document at the end of the treatment, 2, 4 weeks and 7 months clinical improvement of the patient documented by decreasing the EVA score. ${ }^{22}$

In 2008, Murina F. and colleagues carried out a study in 40 patients with vestibulodynia, where they evaluated the efficacy of TENS using a vaginal probe compared to placebo. ${ }^{23}$ Patients in the intervention group received vaginal TENS twice a week at frequencies of $10-50 \mathrm{mHz}$ to complete 20 sessions. The result of the study was a decrease in the score on the viasual scale of pain 2.1 for vaginal TENS versus 5.6 for the placebo group. The follow-up period was short of 3 months after the last treatment session.

Of the few studies that exist on the use of multimodal therapy, Murina and collaborators conducted a study of 20 patients with vestibulodynia where they evaluated the effect of palmitoylethanolamide - transpolidatin combination against placebo, both groups received electro vaginal stimulation in total 7 sessions, they found that the combination of vaginal electro-stimulation plus palmitoylethanolamide - transpolidatin offers better clinical results than using them as monotherapy in patients with vestibulodynia. ${ }^{24}$

The results agree with those thrown in our investigation, however until now there are no established electrostimulation protocols, which would be important to determine in future investigations. On the other hand, given that it is a rare condition, possibly due to the lack of clinical suspicion and approach by the first contact physicians, the studies published so far including the present are samples of small studies.

\section{CONCLUSION}

Chronic pelvic pain is an underdiagnosed disease poorly managed by untrained doctors. Upon reaching our urogynecology clinic with symptoms of more than 6 months of evolution and giving them an adequate diagnosis as well as treatment by multimodal therapy, patients presented a significant improvement without reporting relapses six months after the last electrostimulation session.

The multimodal therapy offered favorable results for all our patients under study and without adverse effects; observing a clinical improvement of $80 \%$ and a progressive decrease of the average EVA score of 7.16 points from the initial evaluation until the twelfth session, ending with an EVA of 2.9 points on average six months after the last session of electro vaginal stimulation.
Finally, patients with chronic pelvic pain of myofascial origin are difficult to treat, with multimodal treatment good clinical results are obtained in these patients. However, greater efforts are needed for a better characterization, diagnostic approach and unification of the different parameters of analgesic electro stimulation in patients with this condition.

\section{ACKNOWLEDGMENTS}

Authors would like to thank to the staff that makes up the gynecological urology clinic of the National Medical Center November 20 of the ISSSTE, for their invaluable support for this study to be carried out.

\section{Funding: No funding sources}

Conflict of interest: None declared

Ethical approval: The study was approved by the Institutional Ethics Committee

\section{REFERENCES}

1. Le PU, Fitzgerald CM. Pelvic Pain: An Overview. Phys Med Rehabil Clin N Am. 2017;28(3):449-54.

2. Ayorinde AA, Macfarlane GJ. Chronic pelvic pain in women: an epidemiological perspective. 2015.

3. Cagnacci A, Vecchia E Della, Xholli A. Chronic pelvic pain improvement: impact on quality of life and mood. Gynecol Endocrinol. 2019:1-4.

4. Spitznagle TM, McCurdy Robinson C. Myofascial pelvic pain. Obstet Gynecol Clin North Am. 2014;41(3):409-32.

5. Yosef A, Ahmed AG, Al-Hussaini T, Abdellah MS, Cua G, Bedaiwy MA. Chronic pelvic pain: Pathogenesis and validated assessment. Middle East Fertil Soc J. 2016;21(4):205-21.

6. Khatri G, Khan A, Raval G, Chhabra A. Diagnostic Evaluation of Chronic Pelvic Pain. Phys Med Rehabil Clin N Am. 2017;28(3):477-500.

7. Quaghebeur J, Wyndaele J-J. Chronic pelvic pain syndrome: Role of a thorough clinical assessment. Scand J Urol. 2015;49(2):81-9.

8. Hunter CW, Stovall B, Chen G, Carlson J, Levy R, Hunter CW. Anatomy, Pathophysiology and Interventional Therapies for Chronic Pelvic Pain: A Review. 2018;(1):147-67.

9. Mieritz RM, Thorhauge K, Forman A, Mieritz HB, Hartvigsen J, Christensen HW. Musculoskeletal dysfunctions in patients with chronic pelvic pain: a preliminary descriptive survey. J Manipulative Physiol Ther. 2016;39(9):616-22.

10. Temme KE, Pan J. Musculoskeletal Approach to Pelvic Pain. Phys Med Rehabil Clin N Am. 2017;28(3):517-37.

11. Bonder JH, Chi M, Rispoli L. Myofascial pelvic pain and related disorders. Phys Med Rehabil Clin N Am. 2017;28(3):501-15.

12. Díaz-Mohedo E, Barón-López FJ, Pineda-Galán C. Etiological, diagnostic and therapeutic consideration 
of the myofascial component in chronic pelvic pain. Actas Urol españolas. 2014;35(10):610-4.

13. Faubion SS, Shuster LT, Bharucha AE. Recognition and Management of Nonrelaxing. JMCP. 2018;87(2):187-93.

14. Borg-Stein J, Iaccarino MA. Myofascial pain syndrome treatments. Phys Med Rehabil Clin N Am. 2014;25(2):357-4.

15. Bartley J, Han E, Gupta P. Transvaginal trigger point injections improve pain scores in women with pelvic floor hypertonicity and pelvic pain conditions. 2018:1-5.

16. Engeler DS, Baranowski AP, Dinis-Oliveira P, et al. The 2013 EAU guidelines on chronic pelvic pain: Is management of chronic pelvic pain a habit, a philosophy, or a science? 10 years of development. Eur Urol. 2013;64(3):431-9.

17. Dommerholt J, Chou L, Finnegan M, Hooks T. A Critical Overview of the Current Myofascial Pain Literature. J Bodyw Mov Ther. 2019.

18. Abello A, Das AK. Electrical neuromodulation in the management of lower urinary tract dysfunction: evidence, experience and future prospects. Ther Adv Urol. 2018;10(5):165-73.

19. Tam J, Loeb C, Grajower D, Kim J, Weissbart S, Tam J. Neuromodulation for chronic pelvic pain. 2018:1-9.
20. Nappi RE, Ferdeghini F, Abbiati I, Vercesi C, Farina C, Polatti F. Electrical Stimulation ( ES ) in the Management of Sexual Pain Disorders Electrical Stimulation ( ES ) in the Management of Sexual Pain Disorders. 2010:37-41.

21. de Oliveira Bernardes N BL. Intravaginal electrical stimulation for the treatment of chronic pelvic pain. $\mathbf{J}$ Reprod Med. 2005;50(4)):267-72.

22. de Bernardes, Marques A, Ganunny C BL. Use of intravaginal electrical stimulation for the treatment of chronic pelvic pain. J Reprod Med. 2010;55(1-2):1924.

23. Murina F, Bianco V, Radici G, Felice R, Martino M Di, Nicolini U. Transcutaneous electrical nerve stimulation to treat vestibulodynia: a randomised controlled trial. 2008:1165-70.

24. Murina F, Graziottin A, Felice R, Radici G, Tognocchi C. Vestibulodynia: Synergy Between. J Low Genit trac Dis. 2013;17:116.

Cite this article as: Reyes GG, Ochoa CB, Marin CMM, Quispe VBA, Barco LED, González AG, et al. Multimodal treatment of myophasal chronic pelvic pain in women: institutional experience. Int $\mathbf{J}$ Reprod Contracept Obstet Gynecol 2019;8:1708-12. 